\title{
Estrategia didáctica para aprendices panameños del idioma chino en estadios iniciales
}

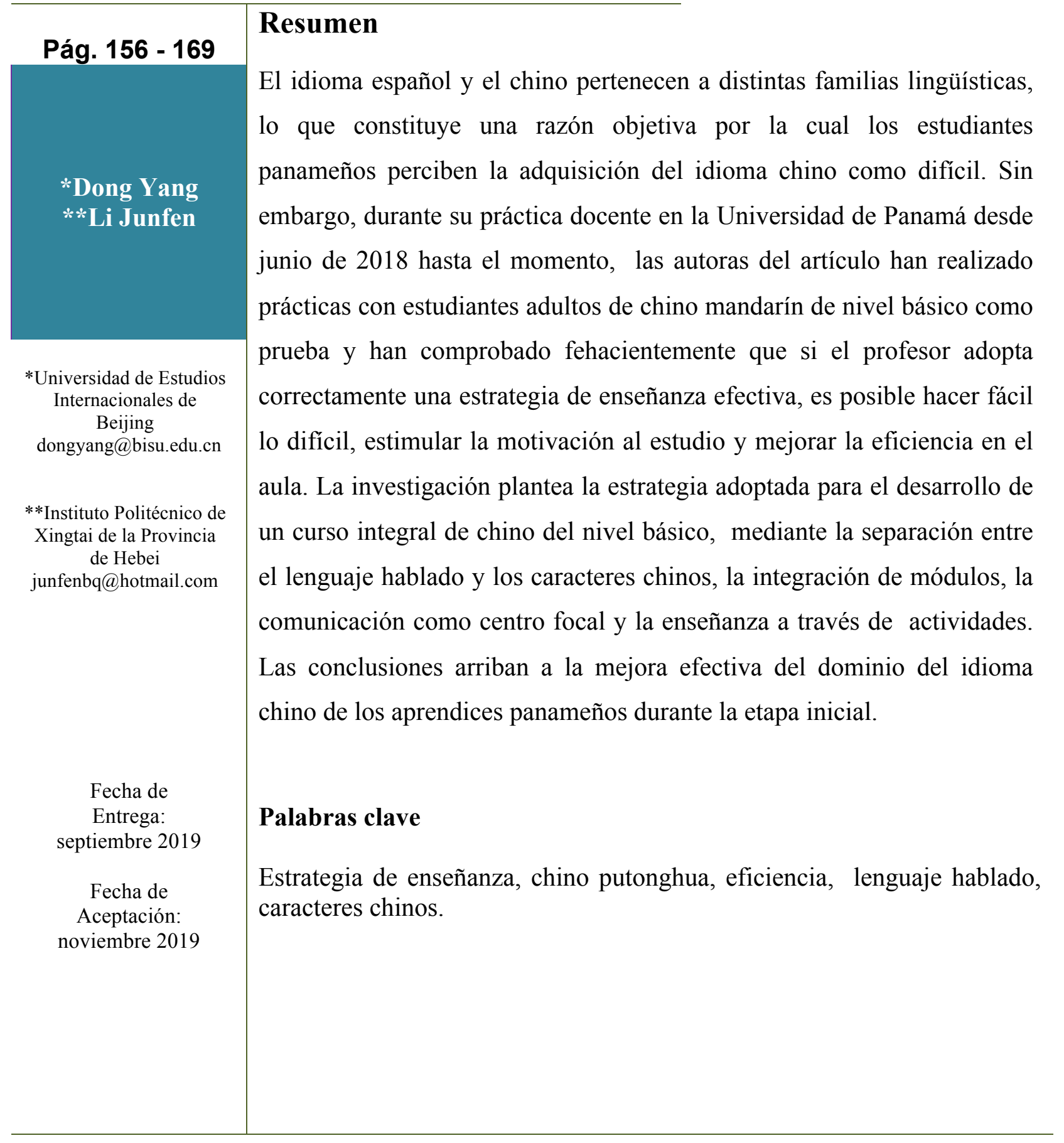




\title{
Teaching strategy for Panamanian learners of Chinese language in early levels
}

\begin{abstract}
Spanish and Chinese belong to different language families, which is the main reason why Panamanian perceive learning Mandarin as a difficult task. Nevertheless, the authors of this article, as a basis, have taught Mandarin Chinese to adult students during their teaching practice at the Universidad de Panama, from June of 2018 up to now, and have reliably proven that, if teachers adopt an effective teaching strategy, it is quite possible to make mandarin learning easier, to stimulate and motivate students to learn and improve classroom efficiency. This research proposes to apply the strategies stated above to develop a comprehensive basic Chinese course, by means of the separation between spoken language and Chinese characters, module integration, communication as a focal point and teaching through activities. We arrive at the conclusion that Panamanian learners have effectively improved their mastery of Chinese language in the initial stage.
\end{abstract}

\section{Key words}

Teaching strategy, Putonghua Chinese, efficiency, spoken language, Chinese characters 


\section{INTRODUCCIÓN}

Aunque el grado de dificultad al que se enfrenta el aprendiz de una lengua extranjera se encuentra condicionado positiva y negativamente por numerosos factores, objetivamente, pivota aquel sobre las cualidades intrínsecas de la propia lengua meta. Si adoptáramos una óptica analítica, podríamos percatarnos de que el germen de la complejidad en el aprendizaje radica en la distancia que media entre la lengua materna y la lengua meta. Así, mientras más puntos en común contengan ambas lenguas, es decir, la distancia que existe entre la lengua materna y la lengua meta sea menor para el estudiante resultará relativamente más fácil la adquisición de la lengua objeto de aprendizaje; por el contrario, cuando la distancia entre ambas resulte mayor, el estudiante atravesará un mayor número de dificultades durante el aprendizaje. Sin embargo, desde el largo plazismo analítico adoptado en nuestra praxis docente del chino, hemos observado que su percepción sobre un idioma extranjero proviene de factores psicológicos subjetivos personales, no existiendo tal supuesta dificultad absoluta. Valga decir como inciso, en este estudio, que cuando hacemos referencia a la lengua china nos estamos refiriendo a la forma estandarizada del chino que es el putonghua, literalmente "lengua común".

Existen dos tipos de factores principales que afectan a la psicología de los estudiantes: factores internos y factores externos. Los factores internos comprenden esencialmente el objetivo al que responde el estudio y la motivación del aprendiz. Los factores externos, por su parte, se encuentran principalmente compuestos por estrategias didácticas correctas y efectivas en diferente grado. Al mismo tiempo, es necesario señalar que, un factor externo también podría impulsar diferentes ciclos vitales de algún factor interno en el estudiante.

El chino pertenece a la familia de lenguas sino-tibetana, mientras que el español pertenece a la familia de lenguas indoeuropeas; en realidad, son dos lenguas sumamente distintas. Por ejemplo: el chino es una lengua tonal, mientras que el español, no; los caracteres chinos son monosílabos, y las palabras en español están constituidas mayormente por dos o más 
sílabas; en términos generales, el chino no depende de la flexión estricta como las lenguas indoeuropeas, sino que principalmente se aprovecha de medios gramaticales como secuencias o partículas para expresar la relación y el significado gramatical, tampoco poseyendo conjugación verbal, ni tiempos, ni concordancia de género y número, etc. Por todo esto, a raíz de tan cuantiosas diferencias, no resulta sorprendente que algunos estudiantes panameños se oculten tras la idea preconcebida de que el chino es muy difícil o extremadamente difícil de aprender.

El proceso de enseñanza y aprendizaje en los estudios iniciales se revela de manera crucial. Sin un buen comienzo, es posible que a los estudiantes les falte motivación, carencia que afectará irremediablemente su aprendizaje. Ante esta situación podríamos preguntarnos: ¿Cómo resolver esa contraproducente predisposición congénita a dar por hecho la enorme dificultad inherente al aprendizaje del chino que podemos percibir en la etapa inicial de adquisición? La respuesta pudiera ser: Sobre la base de la investigación de la diferencia entre los dos idiomas, sobre el pensamiento y los hábitos de estudio de los estudiantes y fortaleciendo la competencia comunicativa en chino como premisa y el uso de la psicología positiva para cubrir la psicología negativa como orientación teórica.

Este artículo pretende explicar las estrategias de enseñanza basadas en la separación entre el lenguaje hablado y los caracteres chinos, la integración de módulos, la comunicación como centro focal y la enseñanza, mediante actividades en la práctica de un curso integral de chino; con el objetivo de potenciar y elevar la autoeficacia, la percepción de éxito y logro, así como el deleite durante el proceso de aprendizaje de los estudiantes. El estudio espera intercambiar opiniones y experiencias con los colegas sobre la enseñanza de idiomas, aportaciones que, sin duda, serán extremadamente valiosas y enriquecedoras.

REVISTA ANUAL ACCIÓN Y REFLEXIÓN EDUCATIVA, N 45 
Estrategia didáctica para aprendices panameños del idioma chino en estadios iniciales

\section{Estrategia didáctica efectiva en la enseñanza del idioma chino.}

La estrategia didáctica efectiva se refiere a los medios y métodos que adoptan los profesores con el objetivo de llegar a la meta de la enseñanza con mayor eficiencia durante las actividades de la enseñanza-aprendizaje.

\section{Desde la perspectiva del modo de enseñanza:}

Debido a las particularidades idiosincrásicas del chino, se puede anotar que, en la práctica docente, dos son los aspectos existentes, esto es el "lenguaje hablado" y los "caracteres". En términos de símbolos lingüísticos, el chino moderno presenta dos sistemas: los caracteres chinos y su sistema de transcripción fonética (pinyin). En los entornos de habla hispana, desde la perspectiva de la efectividad de una enseñanza enfocada hacia la competencia comunicativa en chino, los dos sistemas encierran sus propias ventajas funcionales, poseyendo también, no obstante, deficiencias. En este sentido, organizar científicamente el modo de enseñanza a fin de desarrollar fortalezas y superar debilidades, hacer fácil lo difícil y mejorar la eficiencia, constituyen la base de las estrategias empleadas en una enseñanza efectiva.

En lo fundamental, los caracteres chinos constituyen el sistema simbólico de representación gráfica del idioma chino. Emplearlos directamente como herramienta para la enseñanza del chino como lengua extranjera en un entorno de habla hispana no resulta teóricamente inviable. Sin embargo, en vista de razones objetivas tales como la diferencia entre los caracteres chinos y el alfabeto español o la diferencia entre la velocidad de aprendizaje de dichos caracteres chinos y el entrenamiento de la comunicación oral, podrían generarse rémoras de carácter psicológico evidenciadas en un bajo rendimiento del estudiante y en un lento aprendizaje durante los estadíos iniciales, lo cual causaría un evidente impacto negativo en la enseñanza. Para superar este problema, en la actualidad, la mayoría de los modelos y manuales de enseñanza de chino en el extranjero adoptan una estrategia basada en la separación entre el lenguaje hablado y los caracteres chinos. Primero se enseña el pinyin y luego los caracteres.

REVISTA ANUAL ACCIÓN Y REFLEXIÓN EDUCATIVA, N 45 
En febrero de 1958, la Asamblea Popular Nacional de China aprobó el esquema del alfabeto fonético del chino, denominado Hanyu Pinyin, literalmente "deletreo de los sonidos del chino". Así pues, para la transcripción fonética de los caracteres chinos se adopta el sistema pinyin, un tipo de letras internacionales, fáciles de escribir y leer, que además permiten analizar y describir la pronunciación tonal de manera minuciosa. En total, consta de 26 letras latinas, cuya ordenación también se conforma de acuerdo con el orden tradicional del alfabeto latino, desde la a hasta la $\mathrm{z}$.

Puesto que esas 26 letras no resultan suficientes, se aplican diversos métodos para complementarlas Por ejemplo, la formulación de nuevas combinaciones de letras a través de la unión de dos letras individuales, como "zh, ch, sh y ng”, o el uso de signos adicionales, como el de diéresis en el caso de “ü”. Además, el chino posee otra peculiaridad: es un idioma tonal, donde sus cuatro tonos, junto con el neutro, juegan un papel sumamente importante en la discriminación semántica de morfemas cuasi homónimos. Una pronunciación incorrecta puede provocar malentendidos y confusiones de distinta índole. Este alfabeto fonético es el que se usa en la etapa inicial de adquisición del idioma para conocer la pronunciación de cada nuevo carácter que se aprende. En la enseñanza del chino como lengua extranjera, sin duda, constituye una herramienta auxiliar para el aprendizaje.

Gracias a las nuevas tecnologías informáticas, el pinyin ha facilitado grandemente la escritura en ordenadores o celulares. Con el teclado introducimos el pinyin y en la pantalla aparecen los caracteres chinos de las distintas palabras asociadas a la pronunciación de ese pinyin. Al encontrar el carácter deseado, el usuario lo selecciona y automáticamente aparecerá en la pantalla. Como el progreso tecnológico se sofistica a marchas forzadas, en algunos sistemas más avanzados, al escribir aquellas palabras que resultan más habituales en las formaciones oracionales, basta con escribir la consonante inicial de cada carácter y, automáticamente, aparecerán los caracteres, funcionando igual que la tecnología de "texto predictivo". 
Estrategia didáctica para aprendices panameños del idioma chino en estadios iniciales

En la práctica, el sistema de pinyin ha mejorado significativamente la eficiencia y el desarrollo de las competencias comunicativas en el ámbito de la oralidad, pero nos ha acarreado otro problema: si bien el sistema pinyin efectivamente mejora la competencia expresiva-interactivamediadora en la comunicación oral dentro del proceso docente, cabe destacar que implica una pérdida de la supuesta naturaleza sistemática que ha de revestir una enseñanza consistente de los caracteres chinos. Esto provoca serios retrasos en el avance del aprendiz. En aras de intentar resolver este problema, en la práctica docente, exploramos la estrategia didáctica de separación entre el lenguaje hablado y los caracteres chinos, e integración de módulos.

Cabe señalar que la separación entre el lenguaje hablado y los caracteres chinos pasa por enfrentar objetivamente los atributos esenciales de los caracteres y los atributos de rendimiento del pinyin (Li Xiangping, 2006). Durante las fases embrionarias de un aprendizaje enmarcado en un sistema de enseñanza integral se trazan dos líneas de enseñanza: "utilizar el pinyin para acelerar la comunicación y utilizar los caracteres chinos como base", dos caminos separados pero que, en ocasiones, convergen.

El fomento de las competencias orales y el aprendizaje de los caracteres chinos constituyen dos sistemas diferentes para el estudio y para la estructuración de contenidos. Sin embargo, esta "separación" no posee un carácter absoluto), más bien constituye una sinergia entre el pinyin y los caracteres chinos, junto con la integración de módulos. Durante la enseñanza de la destreza de comprensión auditiva y de expresión oral, con la ayuda de la herramienta del pinyin, se puede mejorar significativamente la sensación de logro de los estudiantes y eliminar esa teórica cognición psicológica innata de los panameños de que el chino es difícil de aprender.

Al mismo tiempo, de acuerdo con las reglas básicas de enseñanza de los caracteres chinos, se establecen cuatro módulos funcionales, que actúan integralmente: aprender la forma del carácter, reconocerlo, saber escribirlo y poder usarlo. El principio que rige la intervención es lo fácil primero, lo difícil después; énfasis en la acumulación y su introducción en el aula, tratando de integrar la enseñanza de los caracteres chinos durante los estadíos más tempranos del 
aprendizaje con la enseñanza de habilidades de comprensión auditiva y expresión oral mediante la herramienta del pinyin, conformando así una estrategia de enseñanza para un curso integral de chino básico acelerando la competencia oral y formando la base de la escritura de los caracteres chinos el espaciado

\section{Desde la perspectiva de la comunicación y las actividades de aprendizaje.}

La estrategia de separación entre el lenguaje hablado y los caracteres chinos, e integración de módulos proporciona garantías básicas para el fructuoso desarrollo del curso integral en sus inicios, pero esto no resulta suficiente. La efectividad de las estrategias de enseñanza también debe pasar por la implementación cuidadosa de métodos de enseñanza específicos, enfatizando la comunicación.

\section{La competencia comunicativa en chino como punto central del proceso de enseñanza.}

La enseñanza del chino en niveles básicos debe postular y alcanzar claramente el objetivo general de desarrollo de la competencia comunicativa en chino, en torno al cual deben gravitar los métodos de enseñanza utilizados. Durante su práctica docente, las autoras han tratado de reforzar la exploración de estrategias de enseñanza efectivas en aspectos relativos a la entonación del habla, la enseñanza de los caracteres chinos y a la propia explicación del profesor.

\section{La enseñanza-aprendizaje de la entonación}

Proporcionar el conocimiento y desarrollar la capacidad de entonación fonética de los principiantes sirve como base para aprender chino por medio de pinyin. En clase, el entrenamiento repetido y la corrección resultan inevitables. ¿Cómo manejar la relación entre el sistema fonético y la competencia comunicativa? ¿Cómo entrenar a los estudiantes, sin que sientan cansancio, generándoles confianza en el aprendizaje? Esto constituye la clave para una 
enseñanza efectiva dentro del aula. La estrategia que hemos explorado es el "método de introducción de comunicación directa", mediante el aprendizaje de palabras a través del pinyin, fortalecimiento del aprendizaje del pinyin a través de -.......continuas palabras y formación de oraciones. Por ejemplo: en el módulo fonético, al aprender las vocales simples de chino "a o e i u", las iniciales "b p m f" y los cuatro tonos, podemos introducir palabras como "papá, mamá, hermano, tío". En el entrenamiento del conocimiento fonético, es cuando se introducen las secuencias comunicativas con estructura oracional: „¡Hola, tío!”, “Gracias mamá!”, “iAdiós hermano!", etc. En el proceso de enseñanza, primeramente se explica a los estudiantes las similitudes y diferencias entre las vocales del español "a e i o u" y del chino "a o e i u", las similitudes y diferencias entre la pronunciación de las iniciales y la de las consonantes del español. Acto seguido; se les facilita a los aprendices la práctica de esos cuatro tonos inexistentes en el sistema fonético del español. Después de eso, en cumplimiento de funciones comunicativas tales como saludos, agradecimientos y despedidas, se integra tanto el entrenamiento de la comunicación como el aprendizaje-consolidación de la entonación del chino.

\section{La enseñanza de los caracteres chinos}

Numerosos profesores suelen enfocarse en la enseñanza de la escritura e interpretación de los caracteres chinos en la etapa inicial, ignorando la consolidación y expansión de la competencia comunicativa dentro de esa comprensión inicial (Sha Zongyuan, 2012). Debido a que el sistema de lecto-escritura de los caracteres chinos y el alfabeto español son muy diferentes, la práctica puede ser difícil para los principiantes. Con vistas al logro y el manejo adecuado de la relación entre el desarrollo de las habilidades de comunicación oral y de la capacidad de reconocimiento, lectura y escritura de los caracteres chinos, dentro de la estrategia docente de un curso integral en etapa inicial, el profesor debería tomar la competencia comunicativa como la tarea central.

Tal como ya se mencionó, la enseñanza de los caracteres chinos en la etapa inicial se integra dentro de la enseñanza de la competencia comunicativa. Se enfoca en la acumulación. y se trata 
de hacer fácil lo difícil. La estrategia no es sino un método de enseñanza paso a paso, de forma progresiva, en donde la comunicación es la base y los caracteres chinos son introducidos por el enseñante paulatina y gradualmente. Es decir, en la práctica docente, continuado primero se practica la expresión oral de acuerdo con el texto, con el objetivo de cumplir la meta del nivel comunicativo. Posteriormente, se pasa al reconocimiento, lectura, escritura y uso de los caracteres chinos. La introducción de estos caracteres, por otra parte, debe seguir la frecuencia de uso, procediendo además según los principios del caracter individual al caracter combinado; de pocos trazos a muchos trazos.

\section{La explicación del docente:}

En clase, el discurso del docente forma parte importante de la estrategia de enseñanza. Más concretamente, constituye la relación entre la explicación y la práctica, revelándose como un factor influyente para lograr el imperativo objetivo comunicativo prefijado (Li Shu, Jiang Liping, 2012). No obstante, en gran cantidad de clases de enseñanza del chino como lengua extranjera o segunda lengua, las explicaciones del profesor ocupan tanto tiempo que los estudiantes no disponen ni remotamente de suficiente tiempo para practicar. Como estos estudiantes no poseen un entorno lingüístico meramente chino o inmersivo (es decir, como si estuvieran en China), la conformación de las habilidades lingüísticas de esos aprendices panameños no puede depender sólo de la explicación del profesor. Así, con el fin de garantizar la efectividad de la capacitación en competencia comunicativa, las autoras elaboramos una fórmula denominada "Ley 20/80", la cual consiste en dividir el período de clase en el 20\% para la explicación del profesor y el $80 \%$, del tiempo para la práctica de los estudiantes.

Al mismo tiempo, teniendo en cuenta las particularidades del español, resultaría ideal que la explicación del profesor fuese formulada en un chino comprensible para los estudiantes, apoyada con fragmentos explicativos en español. Esta estrategia implementada estipula que los profesores en clase tengan que explicar lo más importante y hacer que los estudiantes 
Estrategia didáctica para aprendices panameños del idioma chino en estadios iniciales

practiquen más, asegurando así que las actividades de enseñanza en el aula apunten inequívocamente a la capacitación en competencia comunicativa.

\section{Priorizar las actividades de enseñanza dentro del aula}

En nuestro proceso de investigación y práctica hemos percibido de forma diáfana, que la efectividad de las estrategias didácticas también se ve significativamente influida por los hábitos de aprendizaje de los propios estudiantes. Los aprendices de chino en un entorno de habla hispana presentan unos hábitos de aprendizaje adquiridos en un aula occidental caracterizada por la experiencia de escenario en directo. De acuerdo con nuestras experiencias recopiladas, cuánto más se acerque el método de enseñanza en el aula a los hábitos de aprendizaje del estudiante, mayor eficacia tendrá dicho método. Después de comparar los métodos actuales de enseñanza del chino como lengua extranjera, las autoras llegan a la conclusión de que el método de enseñanza que contiene mayores actividades por parte de los estudiantes es el que alberga más ventajas.

Las actividades en la enseñanza o un enfoque por tareas constituye, de hecho, un método ampliamente utilizado en las aulas de chino en el extranjero en los últimos años, cuyo núcleo consiste en la organización de las sesiones a través de actividades, al mismo tiempo que se completa la impartición teórica y la conformación de la competencia comunicativa. De esta manera, desplazando la atención del profesor al estudiante, se mejora el grado de participación de estos últimos en el aula, aumentando también su interés y la practicidad del aprendizaje que llevan a cabo.

\section{Diseño de actividades en el aula:}

Bajo el concepto de formación integral dentro de la etapa inicial de adquisición de una lengua meta, los profesores primero deberán diseñar cuidadosamente contenidos y actividades de acuerdo con las reglas cognitivas y la base de conocimiento preexistente de los estudiantes. Al organizar actividades, siguiendo el principio de dominar bien el texto y las palabras, luego 
emplear el contenido aprendido de manera integral para el uso de escenarios, los docentes se esfuerzan a la hora de configurar el contenido de la actividad por alcanzar un equilibrio integral entre la forma del idioma chino y la capacidad comunicativa.

Como todos sabemos, en clase, es inevitable que los diversos factores que integran la competencia comunicativa del lenguaje se fragmenten o compartimenten a causa de limitaciones de tiempo y espacio. Por ejemplo: Entonación o fonética, vocabulario, gramática, caracteres chinos; comprensión auditiva, expresión e interacción oral, comprensión lectora, expresión e interacción escrita, traducción, competencia pragmática, competencia intercultural, etc. Resulta obvia la imposibilidad de abordar conjuntamente todas las cuestiones en una sola sesión. En realidad, la mayoría de las clases solo pueden resolver problemas pequeños y parciales.

Es claro que la situación descrita no resulta propicia para la formación de la capacidad comunicativa dentro de una nueva lengua. Por lo tanto, somos de la tesis que las actividades en el aula deben atesorar cuantioso contenido y flexibilidad (Jiang Liping, 2015). Al diseñar las estrategias didácticas para enseñar un idioma, no solo se resaltan el conocimiento teórico y las habilidades perseguidas en esa lección en concreto, sino que también se integran los factores propios de una comunicación realista y contextualizada, a fin de que los elementos que conforman la capacidad comunicativa en una lengua puedan disfrutar de un entrenamiento continuo y equilibrado. Por ejemplo: Comprensión auditiva, expresión oral y reconocimiento de caracteres chinos; funciones comunicativas, estructuras gramaticales e integración cultural, entre otras.

Por otro lado, los profesores deben desempeñar de forma óptima el papel de guías cuando se ejecutan las actividades (Cui Yonghua, 2017). Las instrucciones deben estar claramente definidas y la división de tareas debe realizarse para garantizar que las actividades se lleven a cabo de manera ordenada. Hay que evitar el tipo de situación en la que la clase parece 
animada, pero resulta ineficaz. Los estudiantes que participan en las actividades saben qué están haciendo mientras que los demás, no. Cuando la actividad en cuestión concluya, el profesor deberá propiciar que los estudiantes se autocorrijan y realicen comentarios entre sí para centrar su atención y profundizar en lo expuesto y maximizar su impacto formativo. Finalmente, el profesor es responsable de evaluar el dominio de los aprendices y resumir el contenido de la clase.

\section{Conclusión}

Este artículo explica la estrategia de enseñanza por adoptar dentro de un curso integral de idioma chino del nivel básico como experiencia docente. Se discuten la validez de unas y otras estrategias desde las perspectivas de diferentes modos y métodos, proponiendo la priorización de una estrategia didáctica sustentada en la separación entre el lenguaje hablado y los caracteres chinos, la integración de módulos; la comunicación como el centro focal y la enseñanza mediante actividades claramente definidas y realizadas de manera ordenada.

La práctica docente, orientada mediante la metodología descrita, verifica que dicha estrategia didáctica puede influir de forma positiva en la interiorización de los conocimientos por parte de los estudiantes, y ayudar a los aprendices panameños a superar las enormes diferencias entre los dos idiomas; como también aumentar de manera activa y efectiva su interés en aprender y mejorar su nivel de chino putonghua.

Alberga el presente artículo nuestro deseo de realizar intercambios en torno a esta materia y solicitar los valiosos comentarios de los colegas investigadores. 
Dong Yang y Li Junfen

\section{Referencias}

Cui Yonghua. (2017). Investigación sobre el modelo de enseñanza inmersiva estadounidense Composición y partes del carácter chino. Guangzhou, China: Educación e investigación del chino.

Li Shu, Jiang Liping. (2012). Cómo enseñar chino a extranjeros [M]. Beijing, China: Beijing Language and Culture University Press.

Li Xiangping. (2006). La grafética en la enseñanza de los caracteres chinos [M]. Beijing, China: Language\&Culture Press.

Lin Cuiyun, Su Yanyan. (2007). "Diseño interactivo de cursos artísticos y experimento de valor creativo sobre la base de varias colecciones", Seminario de integración de información en la enseñanza de 2007.

Jiang Liping. (2015). Investigación de modelos de enseñanza mediante actividades. Beijing, China: Chinese Teaching in the World.

Sha Zongyuan. (2012). Una breve discusión sobre el dominio de los caracteres chinos de los profesores de chino y su aplicación en la enseñanza [A]. Teoría de formación de talentos de la enseñanza internacional de chino (3 ${ }^{a}$ serie) $[C]$. Beijing, China: Peking University Press. 